\title{
HispanismeS
}

Revue de la Société des Hispanistes Français

\section{Quand les liens de famille troublent la fête}

Éléments pour une lecture de la nouvelle « Feliz aniversário » de Clarice Lispector

Cuando los lazos familiares perturban la fiesta. Elementos para la lectura del cuento "Feliz aniversário" de Clarice Lispector

When the family ties disturb the party. Elements for a reading of the short story "Feliz aniversário" by Clarice Lispector

\section{Mireille Garcia}

\section{(2) OpenEdition}

\section{Journals}

Édition électronique

URL : https://journals.openedition.org/hispanismes/570

DOI : $10.4000 /$ hispanismes. 570

ISSN : 2270-0765

\section{Éditeur}

Société des Hispanistes Français

\section{Référence électronique}

Mireille Garcia, "Quand les liens de famille troublent la fête », Hispanismes [En ligne], 15 | 2020, mis en ligne le 01 juin 2020, consulté le 31 juillet 2021. URL : http://journals.openedition.org/hispanismes/ 570 ; DOI : https://doi.org/10.4000/hispanismes.570

Ce document a été généré automatiquement le 31 juillet 2021.

Les contenus de cette revue sont mis à disposition selon les termes de la Licence Creative Commons Attribution - Pas d'Utilisation Commerciale - Pas de Modification 4.0 International. 


\section{Quand les liens de famille troublent la fête}

Éléments pour une lecture de la nouvelle « Feliz aniversário » de Clarice Lispector

Cuando los lazos familiares perturban la fiesta. Elementos para la lectura del cuento "Feliz aniversário" de Clarice Lispector

When the family ties disturb the party. Elements for a reading of the short story

"Feliz aniversário" by Clarice Lispector

\section{Mireille Garcia}

\section{Introduction}

1 «La fiction de Clarice a élaboré un mode de pensée très spécial»; tel est le commentaire qui figure sur la couverture intérieure de l'ouvrage Clarice Lispector, uma literatura pensante ${ }^{1}$. Ainsi communiquée d'emblée au lecteur, la complexité de l'œuvre lispectorienne pose la question de savoir comment lire Clarice Lispector, car « elle appartient [...] à cette lignée d'auteurs dont l'œuvre défie la littérature, ses catégories, ses codes. Elle a avec la "République des Lettres" une relation ambivalente, tendue sinon conflictuelle $»^{2}$. Aussi, avant d'entreprendre notre étude, il nous semble nécessaire de réaliser quelques incursions théoriques au sujet de l'écriture lispectorienne afin d'en relever les principaux questionnements jugés pertinents à l'analyse de cette nouvelle.

2 Vaste est la fortune critique de Clarice Lispector, romancière dont l'œuvre fait aujourd'hui l'objet de grand nombre d'études tant au Brésil qu'à l'étranger. Mais cela n'a pas toujours été le cas : alors que son premier roman est publié en 1943, ce n'est qu'à partir des années 1960 que sa production commence à susciter l'intérêt de la critique $^{3}$, «l'obligeant à revoir sa perspective $»^{4}$. Parmi les théoriciens et auteurs qui ont accompli des travaux pionniers et essentiels à son sujet, le critique littéraire nordaméricain Benjamin Moser et l'écrivaine française Hélène Cixous publient des ouvrages 
sur la romancière contribuant à la diffusion de son œuvre à l'international. Au Brésil, c'est sans aucun doute Benedito Nunes qui s'érige en spécialiste de l'auteure aux côtés de Nadia Batella Gotlib, Olga Borelli ou encore Evando Nascimento pour ne citer qu'eux $^{5}$. Tous s'accordent à dire que Clarice Lispector démonte les schémas narratifs traditionnels inaugurant une "littérature pensante » où l'introspection, l'observation et le langage donnent à son écriture un caractère de confession orale. La dimension psychologique réside dans le fait que ses écrits - tous autant qu'ils sont - abordent une "thématique manifestement existentielle " ${ }^{6}$. Le recueil de nouvelles Laços de família $(2009)^{7}$ ne déroge pas à la règle avec des récits rythmés par un «flux de conscience » et une "quête des sens ${ }^{8}$ et dans lesquels prévalent des instants de "tension conflictuelle », de « dévoilement de l'existence » ou encore de "puissance magique du regard $»^{9}$, autant d'éléments qui révèlent une nouvelle conception du monde suggérée par la "littérature pensante" de Clarice Lispector. Dès lors, un langage à la fois sensoriel et pictural sillonne l'œuvre ouvrant la voie à une nouvelle expression verbale intense que l'auteure elle-même déclare être une " fête des mots » ${ }^{10}$.

C'est précisément dans le cadre de la fête que nous place d'emblée la lecture de la nouvelle «Feliz aniversário». Comprise comme un «ensemble de réjouissances collectives destinées à commémorer périodiquement un événement $»^{11}$, la fête est un objet d'étude récurrent dans les domaines de l'histoire, de l'anthropologie et de la sociologie, mais tient également une place de choix dans diverses productions littéraires qui, bien souvent, lui confèrent un rôle au-delà de la simple activité sociale : elle devient, dans le même temps, espace physique et symbolique, produit de continuité et de rupture, lieu d'instauration d'un ordre et d'un désordre social ou encore scène privilégiée des tensions et des conflits familiaux. C'est par la fête que cette cinquième nouvelle du recueil Laços de familia - dont le titre laisse supposer qu'une réflexion sur les rapports familiaux traverse toute l'œuvre - met en scène les principaux contours du conflit familial : loin d'être vécue comme un temps de partage et de convivialité, la fête est ici troublée par ces liens de famille qui se révèlent être davantage des liens qui musellent et emprisonnent plutôt qu'ils n'unissent et ne rassemblent. Procédant volontairement - et de façon ironique - à une inversion du paradigme, Clarice Lispector met en scène cette fête d'anniversaire comme un espace empli de significations où les éléments festifs se dissipent pour laisser place à une ambiance oppressante et révélatrice tant de la dynamique structurelle de la famille que du contexte socioculturel dans lequel elle s'insère. À mesure que le lecteur accompagne la fête qui se met en place, se déroule puis s'achève, il est également invité à arpenter progressivement l'univers lispectorien dans lequel la « dimension scopique $»^{12}$ et l'importance du regard mènent à l'éclosion ou la révélation d'un " événement $~^{13}$ pour enfin revenir presque au point de départ ${ }^{14}$. C'est à partir de ces considérations que ce travail interrogera la fonction que tient la fête dans la nouvelle choisie, ainsi que les relations familiales qui y sont dépeintes. Pour ce faire, nous prendrons comme fil conducteur de notre réflexion les éléments mis en avant dans l'étude de Benedito Nunes qui nous fournit les clés permettant de saisir les significations profondes de la nouvelle. 


\section{Que la fête commence : I'anniversaire comme espace privilégié des « liens » de famille et de l'observation contemplative}

4 Adapté à deux reprises - en 1977 puis en 1994 - sur la chaîne de télévision la plus populaire du Brésil, et par la suite largement exploité dans les programmes scolaires de l'enseignement secondaire, la nouvelle «Feliz aniversário » est sans doute l'une des plus mémorables du recueil. Supposément triviale et ordinaire, la trame de cette nouvelle décrit la fête d'anniversaire d'une aïeule en présence de nombreux membres de sa famille réunis pour l'occasion ; telle une tradition, la fête d'anniversaire est avant tout une réunion familiale censée renforcer les liens de parenté autour d'un rite de passage. Toutefois, l'histoire ici ne tarde pas à révéler la complexité des rapports et des choses qui la composent, étant donné que ce qui transparaît en premier lieu est le conflit qu'alimentent tous les personnages les uns envers les autres. En effet, les rapports conflictuels se manifestent dès l'ouverture de la nouvelle qui débute par l'arrivée des convives à la fête : les dissensions affleurent alors même que les festivités n'ont pas commencé. Ainsi, la bru d'olaria se présente sans son mari : « 0 marido não veio por razões óbvias : não queria ver os irmãos. Mas mandara sua mulher para que nem todos os laços fossem cortados» (p. 54). Si les membres de la fratrie semblent entretenir des rapports conflictuels, il en est de même pour les belles-filles qui alimentent mutuellement des rancœurs: "[...] a nora de Ipanema pensou que não suportaria nem um segundo mais a situação de estar sentada defronte da concunhada de Olaria - que cheia das ofensas passadas não via um motivo para desfitar desafiadora a nora de Ipanema. » (p.56). Force est alors de constater que les conflits familiaux décrits sont des éléments qui soulignent la déconstruction opérée par l'auteure par rapport à un ordre établi : l'anniversaire - fête familiale par excellence - se caractérise davantage par les rivalités et les conflits alors que la fête est supposément le moment d'une grande fraternité retrouvée. Ainsi présentée, elle est dès lors une occasion de rupture et de désunion familiale que l'on peut notamment percevoir dans l'attitude des convives qui, comme contraints et forcés, se doivent d'accomplir leur obligation familiale. C'est ainsi que sans détours la bru d'Olaria déclare : «Vim para não deixar de vir» (p. 54), et qu'à l'issue de la fête «[...] com alívio os convidados se encontraram na tranquilidade fresca da rua» (p. 66). Par ailleurs, d'aucuns négligent les convenances qui exigent qu'on ne vienne pas les mains vides, et tous semblent remettre en cause l'utilité d'une telle célébration :

Então, como se todos tivessem tido a prova final de que não adiantava se esforçarem, com um levantar de ombros de quem estivesse junto de uma surda, continuaram a fazer a festa sozinhos, comendo os primeiros sanduíches de presunto mais como prova de animação que por apetite, brincando de que todos estavam morrendo de fome (p. 57).

Dans le même ordre d'idée, Zilda - la maîtresse de maison - se montre vindicative à l'encontre de ses belles-sœurs puisqu'aucune ne lui propose son aide pour l'organisation de la fête: "[...] nenhuma cunhada ajudou propriamente» (p. 57). Endossant le rôle de serviteur chargée de l'intendance, Zilda sait que le poids de la fête repose tout entier sur elle et éprouve un sentiment à la fois d'angoisse et de révolte visà-vis des membres de sa famille qui n'expriment aucune reconnaissance ni gratitude à son égard: 
Mas ninguém elogiou a ideia de Zilda, e ela se perguntou angustiada se eles não estariam pensando que fora por economia de velas - ninguém se lembrando de que ninguém havia contribuído com uma caixa de fósforos sequer para a comida da festa que ela, Zilda, servia como uma escrava, os pés exaustos e o coração revoltado (p. 58). la relation qu'elle maintient avec sa mère - «a aniversariante » (p. 54) : se hâtant d'en finir avec ce qui semble être une contrainte :

Zilda [...] arrumara a mesa cedo [...] enfeitara a mesa logo depois do almoço [...] E, para adiantar o expediente, vestira a aniversariante logo depois do almoço. Puseralhe desde então a presilha em torno do pescoço e o broche, borrifara-lhe um pouco de água-de-colônia para disfarçar aquele seu cheiro de guardado - sentara-a à mesa. E desde as duas horas a aniversariante estava sentada à cabeceira da longa mesa vazia, tesa na sala silenciosa (p. 55).

7 Telle une mise en scène hypocrite - où les prétendus liens affectifs se transforment en liens qui musellent à l'image de la "presilha em torno do pescoço " de l'aïeule, semblable à un collier de chien -, l'organisation et l'espace de la fête révèlent ainsi les tensions familiales et ce, par le biais d'un narrateur-observateur qui se place en dehors de l'histoire mais à l'intérieur du discours, révélant un procédé narratif élaboré que Benedito Nunes qualifie de « régime de transaction » ou mode alternatif de narration:

A romancista, que adota a terceira pessoa, não se suprime como instância externa da narração. Mas também percebe e sente com a personagem. Ora a ela aderindo, ora lhe impondo a sua presença como sujeito-narrador, a romancista pratica um modo de ver oscilante, verdadeiro regime de transação, que se reflete na alternância do discurso direto e do indireto [... $]^{15}$.

8 Cette technique de narration ou «stratégie générale de sa logique narrative dissimulatrice ${ }^{16}$, comme la qualifie Nadia Batella Gotlib, est en réalité une manière pour l'auteure de construire son récit autour du regard, lui conférant ainsi une dimension "scopique» à l'allure d'un kaléidoscope ou d'un patchwork fait des fragments et des bribes de discours et pensées de chacun des personnages qu'il observe. Ainsi, le narrateur adopte la technique de l'enchevêtrement de voix et de sujets qui résulte en un amas de personnages pour la plupart sans nom ${ }^{17}$, et que seules quelques brèves descriptions physiques et psychologiques permettent d'identifier. De sorte que belles-sœurs, brus, enfants et petits-enfants, nièces et cousines forment le panel de cette famille désunie et manifestement incapable de communiquer, réunie à l'occasion d'un anniversaire supposément joyeux mais dans un climat tendu, fallacieux et tumultueux.

C'est au centre de ce tumulte faussement festif que se trouve celle dont on fêtait ce jour-là l'anniversaire, "a aniversariante » (p.54), qui se voit attribuer de nombreux noms d'emprunts tels que "a mãe», "mamãe», "a velha», "vovó» ou encore "vovozinha ", avant que son nom - dona Anita - finisse par enfin être révélé, laissant supposer là encore une forme de dépersonnalisation voire de spectralité, phénomène que Baudrillard définit comme étant la «résonnance d'un réel qui s'estompe, qui devient fantomatique ${ }^{18}$. En effet, sa présence fantomatique réside dans le fait qu'elle semble être absente et figée, murée dans un silence incompréhensible comme en témoigne le passage suivant: "Os músculos do rosto da aniversariante não a interpretavam mais, de modo que ninguém podia saber se ela estava alegre. Estava era posta à cabeceira. Tratava-se de uma velha grande, magra, imponente e morena. Parecia oca » (p. 56). Le narrateur mentionne à deux reprises que la vieille ne bronchait 
pas: "A velha não se manifestava» (p.56 et 57), insistant sur son silence qui, interprété comme "prélude d'ouverture à la révélation » ${ }^{19}$, peut ici «s'imposer pour que quelque chose se dévoile», capable de transmettre un message et ainsi constituer un langage à part entière. Si le silence s'avère être la forme d'expression d'un langage, il en est de même pour le regard, qui tout aussi symbolique "est l'instrument des ordres intérieurs : il tue, fascine, foudroie, séduit, autant qu'il exprime [...] (et) apparaît comme le symbole et l'instrument d'une révélation ${ }^{20}$. L'observation contemplative de l'aïeule qui, dans une angoisse muette « acompanhava, fascinada e impotente, o voo da mosca em torno do bolo» (p.56) traduit ce que Nunes appelle le «dévoilement silencieux ", situation qui fait en sorte que «les objets exercent une fascination [...] s'insinuant à l'expérience interne lors de moments de pause contemplative $»^{21}$.

10 Ainsi, la crise s'amorce tout en silence et en contemplation, alors que les convives bruyants et agités prétendent avoir faim, feignent être joyeux et usent d'un faux langage, autant de signes qui démystifient les rituels et les codes propres à la fête. Telle une mascarade où les rapports et attitudes de chacun sont théatralisés, la fête est dès lors représentée comme un microcosme de la société, comme un espace psychologique et physique qui réunit les appartenances sociales pour mieux les séparer. En ce sens, elle devient un espace social dans lequel les conflits individuels et internes se confondent avec les différences collectives et sociales, mais aussi la scène d'une révélation, d'une tension conflictuelle qui dénonce le contexte dans lequel s'insère cette famille, à savoir, le patriarcat.

\section{La fête bat son plein : l'anniversaire comme révélateur des liens sociaux et conflictuels}

11 D'après une conception communément répandue, la fête est l'espace de la fusion des classes, autrement dit un espace communautaire où les distinctions sociales sont abolies ne serait-ce qu'un court instant, donnant l'illusion d'une société unie par les codes. Loin de recréer l'image d'une société idéale, la fête d'anniversaire de Clarice Lispector ne fait au contraire qu'accentuer les écarts et les divergences sociales, dénonçant par là-même un modèle de société aux caractéristiques patriarcales et aux tendances conservatrices. C'est pourquoi on note par exemple l'importance du détail dans la description de certains personnages vêtus de leurs plus beaux atouts pour l'occasion, à l'instar de la bru d'Olaria : " esta vinha com o seu melhor vestido [...] com enfeite de paetês e um drapeado » (p.54), le tout dans le seul but de montrer qu'elle n'avait pas besoin d'eux : «[...] não precisava de nenhum deles » (p. 54). C'est elle aussi qui se pose en véritable critique, jugeant l'apparence des autres convives:

[...] ela analisava crítica aqueles vestidos sem nenhum modelo, sem um drapeado, a mania que tinham de usar vestido preto com colar de pérolas, o que não era moda coisa nenhuma, não passava era de economia (p. 62).

12 Et ce n'est pas un hasard si, en l'absence de nom, la seule information qui nous est donnée à connaître pour ce personnage est son quartier de provenance : Olaria ${ }^{22}$. Car il s'avère en réalité que l'espace de la fête prend des allures de "géographie sociale », où des quartiers sont socialement identifiés, ce qui accentue le contraste et les disparités sociales - et par conséquent les relations - comme en témoigne l'étude de Roberto Corrêa dos Santos : 
A própria seleção dos bairros [...] serve de cenário e estabelece uma geografia social através da qual os contrastes se reforçam. Os três bairros cariocas mencionados Olaria, Copacabana e Ipanema - não são marcados sem intenção; destacam os três diferentes espaços sociais da cidade, exemplificativos e metonímicos em relação às diferentes economias. Tais bairros, segundo o texto, são caracterizados pelas ideologias de seus respectivos representantes ${ }^{23}$.

13 Dans le même ordre d'idée, l'agencement de l'espace de la fête - où la maîtresse de maison «[havia] disposto cadeiras unidas ao longo das paredes » (p.54) - est lui aussi révélateur de la fracture sociale entre les membres de la famille qui, se faisant face, s'affrontent du regard ou à l'inverse se dérobent, à l'instar de la bru d'Ipanema : « [...] na fila oposta das cadeiras fingindo ocupar-se com o bebê para não encarar a concunhada de Olaria » (p.55). Toujours dans le cadre de la représentation sociale au sein de la fête, anglicismes et allusions à la culture et à la langue anglaise témoignent eux aussi des disparités sociales : l'« American Way of Life » ${ }^{24}$ s'invite à la fête comme en témoignent les ballons sur lesquels était écrit «Happy Birthday », le Coca-Cola sur la table, les petits-enfants du Collège Bennett ou encore la bonne Dorothy dont le nom à consonance étrangère est plus qu'évocateur. Signe extérieur à la fois d'une modernité et d'un certain statut, cette américanisation ne fait qu'accentuer le déphasage dans lequel se trouve la famille, comme on peut le lire dans le passage suivant:

Como não haviam combinado, uns cantaram em português e outros em inglês.

Tentaram então corrigir: e os que haviam cantado em inglês passaram a português,

e os que haviam cantado em português passaram a cantar bem baixo em inglês

(p. 58).

14 Tout ceci reflète également la réflexion critique qu'élabore la romancière sur les normes de comportement et la place destinée à la femme dans l'ordre social et culturel. En effet, la représentation de l'ordre patriarcal est soulignée tout particulièrement en la personne de Zilda, "a única mulher entre os seis irmãos homens e a única que, estava decidido já havia anos, tinha espaço e tempo para alojar a aniversariante » (p. 55), celle qu'on qualifie de maîtresse de maison - « a dona de casa » (p. 55) - , celle à qui incombe la lourde tâche d'organiser la fête et qui ne semble pouvoir évoluer dans un autre espace que celui de l'intérieur de l'appartement, entre la cuisine et le salon. Soumise et tributaire, elle dénonce de manière figurative l'assujettissement de la femme au sein de la société brésilienne patriarcale de l'époque, mortifiée à l'idée d'être jugée :

[...] não queria sequer olhar os outros, sabia que os desgraçados se entreolhavam vitoriosos como se coubesse a ela dar educação à velha, e não faltaria muito para dizerem que ela já não dava mais banho na mãe, jamais compreenderiam o sacrifício que ela fazia (p. 61).

C'est justement dans ce cadre que se produit la tension conflictuelle, à savoir le moment culminant de la crise, l'instant de la révélation d'un événement : alors que la fête bat son plein et qu'arrive l'heure de couper le gâteau, soudainement l'aïeule empoigne le couteau : «[...] de súbito a velha pegou na faca. E sem hesitação [...] deu a primeira talhada com punho de assassina » (p. 59). S'en suit presque immédiatement le deuxième "événement » lorsqu'elle cracha par terre : "Incoercível, virou a cabeça e com força insuspeita cuspiu no chão » (p. 60). Transportée par un sentiment puissant de haine et de colère qui explose soudainement, le personnage, sujet à une brusque transformation, personnifie ce que Nunes appelle la tension conflictuelle :

Na maioria dos contos da autora, o episódio único que serve de núcleo à narrativa é um momento de tensão conflitiva [...] [que] se declara subitamente e estabelece uma 
ruptura do personagem com o mundo. Noutros porém a crise declarada [...] mantém-se do princípio ao fim, seja como aspiração ou devaneio, seja como malentendido ou incompatibilidade entre pessoas, tomando a forma da estranheza diante das coisas, do embate dos sentimentos ou de consciência culposa ${ }^{25}$.

Si l'on en croit la théorie de Nunes, le moment de crise intérieure est conditionné par une situation de confrontation, dans le cas présent une prise de conscience ou la constatation d'une vérité, d'une réalité vis-à-vis de son existence et de sa famille. Aussi, c'est le mépris qu'elle éprouve pour les siens qui déclenche brusquement sa clairvoyance :

Todos aqueles seus filhos e netos e bisnetos que não passavam de carne de seu joelho, pensou de repente como se cuspisse [...] Oh o desprezo pela vida que falhava. Como?! como tendo sido tão forte pudera dar à luz aqueles seres opacos, com braços moles e rostos ansiosos? Ela, a forte, que casara em hora e tempo devidos com um bom homem a quem, obediente e independente, ela respeitara; a quem respeitara e e que lhe fizera filhos e lhe pagara os partos e lhe honrara os resguardos. 0 tronco fora bom. Mas dera aqueles azedos e infelizes frutos, sem capacidade sequer para uma boa alegria. Como pudera ela dar à luz aqueles seres risonhos, fracos, sem austeridade? O rancor roncava no seu peito vazio. Uns comunistas, era o que eram; uns comunistas. Olhou-os com sua cólera de velha. Pareciam ratos se acotovelando, a sua família (p. 60).

17 Le simple regard qu'elle porte sur les siens déclenche une impulsion d'agressivité et de destruction, exprimant une violence intériorisée qui, dans un accès de rage silencieuse, la pousse à renier et à mépriser cette famille qui est la sienne. En accord avec un discours conservateur et autoritaire, elle va jusqu'à associer ses propres enfants à des communistes - terme qui dénote un discours social révélateur des tensions politiques de l'époque - faisant montre d'une férocité presque animale et instinctive qui se reflète aussi dans ses propos lorsqu'elle compare à deux reprises les membres de sa famille à des rats, les percevant comme étant plus proches de l'animalité que de l'humanité :

Pareciam ratos se acotovelando, a sua família. Os meninos, embora crescidos provavelmente já além dos cinquenta anos, que sei eu! - os meninos ainda conservavam os traços bonitinhos. Mas que mulheres haviam escolhido! E que mulheres os netos - ainda mais fracos e mais azedos - haviam escolhido. Todas vaidosas e de pernas finas, com aqueles colares falsificados de mulher que na hora não aguenta a mão, aquelas mulherezinhas que casavam mal os filhos, que não sabiam pôr uma criada em seu lugar, e todas elas com as orelhas cheias de brincos nenhum de ouro! A raiva a sufocava (p. 61).

Conditionnée par un état de soumission déterminé par l'idéologie conservatrice dominante, elle transporte le lecteur à l'époque des patriarches, reproduisant mentalement un discours misogyne qu'elle verbalise finalement en prononçant des propos injurieux : «Que o diabo vos carregue, corja de maricas, cornos e vagabundas!» (p. 62). L'acte de verbaliser choque les convives mais ces derniers ne réagissent pas: "Todos tinham ficado cegos, surdos e mudos [...] olhavam impassíveis [...] todos se entreolharam polidos, sorrindo cegamente, abstratos [...] com estoicismo, recomeçaram as vozes e risadas" (p.62) alors que l'aïeule silencieuse reprend sa contemplation passive : "Seu olhar estava fixo, silencioso. Como se nada tivesse acontecido» (p. 62). Les sentiments d'étrangeté ${ }^{26}$ et d'incompréhension ici à leur paroxysme vont précipiter la fin de la rencontre et des prétendues festivités. C'est sur cette note que la fête s'interrompt d'abord, puis prend fin : «A festa interrompida [...] E por assim dizer, de novo a festa estava terminada » (p. 62 et p. 63). 


\section{La fête est finie : l'anniversaire comme la célébration de la mort ou la transition régressive}

19 La fête, on l'a vu, n'est ici qu'apparences et faux-semblants, scène privilégiée des conflits familiaux et sociaux. Dès lors, nous sommes en droit de nous demander si l'on peut encore parler de fête alors que les événements qui nous sont donnés à voir semblent avoir pour seule vocation celle de déconstruire et de désarticuler les liens. Toutefois, même rompus, ces liens doivent être maintenus afin de ne pas mettre en péril la continuité du système familial, car la ruine de la famille figurerait aussi celle de tous ses membres. La fête a donc bien lieu, mais la commémoration ne répond en réalité qu'à un rite qu'il faut respecter, cette étape qui jalonne la vie et permet le passage à un autre âge : l'anniversaire. Autrement dit, l'anniversaire ici présuppose davantage la mort que la commémoration de la vie. C'est dans ce sens que l'étude de Jean-Claude Schmitt se propose d'apporter une réflexion sur le rôle de l'anniversaire et ses diverses figurations à travers les époques :

[...] pendant tout le «long Moyen Age » (et jusqu'à aujourd'hui dans la langue liturgique), anniversarium et souvent même dies natalis ne désignent pas l'anniversaire de la naissance, mais celui du jour de la mort. C'est ce jour qui seul importait en vérité, celui de la "vraie naissance», de l'entrée par la mort dans la « vraie vie » de l'au-delà et du salut tant espéréz ${ }^{2}$.

20 À la lumière de ces considérations, il apparaît clairement que dans cette nouvelle, la question de la vieillesse et de la mort est aussi explicite que celle de la naissance de la vie suggérée par le titre. Evoquées à diverses reprises et de façon métonymique, la vieillesse et la mort se lisent dans le personnage de l'aïeule et dans les éléments propres à la fête qui l'entourent. Ainsi, «o bolo apagado, grande e seco » (p. 58) qu'elle observe, tout comme " os farelos secos » (p. 59) ou encore « [...] a toalha manchada de Coca-Cola [e] o bolo desabado » (p.60) devant lesquels elle se trouve assise traduisent sa propre décrépitude. Par ailleurs, une allusion à la mort, voire même à l'inhumation, est aussi manifeste dans le geste du partage du gâteau distribué « dada a primeira talhada, como se a primeira pá de terra tivesse sido lançada » (p. 59). L'allusion à la mort se lit aussi dans les réflexions de certains membres de la famille qui se posent des questions: «pensavam se a velha resistiria mais um ano » (p. 67), comme nourrissant l'espoir de ne pas avoir à revivre encore cette fête, bien que la vieille semble s'arranger pour rester en vie : «[...] a artimanha da velha que espertamente sempe vivia mais um ano » (p. 65).

21 Toutefois, comme le suggère l'étude de Schmitt, le jour de la mort est aussi celui de la vraie naissance ${ }^{28}$, et c'est dans cette optique que le moment de tension conflictuelle vécu par l'aïeule - à savoir la révélation suscitée par sa soudaine clairvoyance - peut être interprété. De fait, la scène qui suit le moment de crise rapporte que les membres de la famille s'accordaient à dire que la vieille était tombée en enfance: "[...] eles abanavam a cabeça como se estivessem de acordo que a velha não passava agora de uma criança » (p. 61). Tel un rite de passage, «l'expérience de la crise passe par une transition régressive, c'est-à-dire que l'on redevient un enfant $»^{29}$. Et ce phénomène que l'on peut percevoir comme une renaissance est corroboré par la fête elle-même, ou plutôt, par ses éléments de décoration aux allures de fête pour enfant comme en témoignent les passages suivants: "[...] guardanapos de papel colorido e copos de papelão alusivos à data [...] balóes sungados pelo teto em alguns dos quais estava escrito 
“Happy Birthday!", em outros “Feliz aniversário!". No centro [...] o enorme bolo açucarado » (p.55). Ainsi métamorphosée, l'aïeule passe par une transformation intérieure, insaisissable et aux yeux de tous, mais pourtant effective :

[...] ninguém poderia adivinhar o que ela pensava [...] a aniversariante era apenas o que parecia ser: sentada à cabeceira da mesa imunda, com a mão fechada sobre a toalha como encerrando um cetro, e com aquela mudez que era a sua última palavra. Com um punho fechado sobre a mesa, nunca mais ela seria apenas o que ela pensasse. Sua aparência afinal a ultrapassara e, superando-a, se agigantava serena (p. 64). correspondrait à ce qu'il qualifie être le «dévoilement de l'existence ", à savoir cet « instant précis qui indique le climax de la trame $»^{30}$. C'est précisément au cours de ce bref instant que l'on assiste au seul véritable échange, à la seule communication et au seul lien présent dans la nouvelle entre l'aïeule et une de ses brus, Cordélia :

Cordélia olhou-a espantada. 0 punho mudo e severo sobre a mesa dizia para a infeliz nora que sem remédio amava talvez pela última vez: É preciso que se saiba. É preciso que se saiba. Que a vida é curta. Que a vida é curta. Porém nenhuma vez mais repetiu. Porque a verdade era um relance. Cordélia olhou-a estarrecida. E, para nunca mais, nenhuma vez repetiu - enquanto Rodrigo, o neto da aniversariante, puxava a mão daquela mãe culpada, perplexa e desesperada que mais uma vez olhou para trás implorando à velhice ainda um sinal de que uma mulher deve, num ímpeto dilacerante, enfim agarrar a sua derradeira chance e viver. Mais uma vez Cordélia quis olhar. Mas a esse novo olhar - a aniversariante era uma velha à cabeceira da mesa. Passara o relance (p. 64).

Ce passage souligne la dimension métaphysique dans laquelle s'insère la nouvelle, à savoir les questionnements sur le sens de la vie, de la mort et de l'existence. Il s'agit d'un bref instant décisif de révélation et d'éclosion d'une vérité, sorte de confession qui dénote un élan de transgression du personnage qui se place dans une position inverse à celle à laquelle elle adhère quotidiennement: comme affranchie du schéma de domination, sa pensée semble avoir évolué pour aboutir à son indépendance. Aussi, l'espoir, l'amour, la pleine conscience des faits qui jusqu'alors étaient inexistants dans ce récit, se manifestent brièvement à l'adresse de Cordélia, seul personnage qui n'est pas dépourvu d'identité, et pour cause : elle est la représentation symbolique de l'aïeule et, au même titre qu'elle, demeure dans une position contemplative qui marque sa nonappartenance à la famille.

\section{Conclusion}

En ce qui concerne son processus de création pour certains de ses écrits, Clarice Lispector a fait quelques commentaires et réflexions qu'elle-même a qualifiés comme étant une « explication inutile », et qui figurent dans l'œuvre A legião estrangeira, sous le nom de Fundo de Gaveta (fond de tiroir). Au sujet de la nouvelle « Feliz aniversário », elle écrit :

O que me lembro do conto «Feliz aniversário ", por exemplo, é da impressão de uma festa que não diferente de outras de aniversário; mas aquele era um dia pesado de verão, e acho até que nem pus a ideia de verão no conto. Tive uma «impressão » de onde resultaram algumas linhas vagas, anotadas apenas pelo gosto e necessidade de aprofundar o que se sente. Anos depois, ao deparar com essas linhas, a história inteira nasceu, com uma rapidez de quem estivesse transcrevendo cena já vista - e no entanto nada do que escrevi aconteceu naquela ou em outra festa $[. . .]^{31}$. 
Si on retrouve bien là les caractéristiques du flux de conscience et de la pensée-écriture propres à l'auteure, il est difficile de croire que cette fête d'anniversaire n'a pas été différente des autres. Car l'art de manier la parole et le silence, le regard et les sentiments, font de ce texte en apparence banal, un texte profond et empli de significations qu'il faut savoir lire. Et c'est bien là que réside toute la complexité : savoir lire Clarice. Selon Nadia Gotlib, « lire Clarice serait [...] découvrir comme cela se passe, en chacun de ses textes, en identifiant les manières de miner un territoire apparemment calme, en le faisant sortir des profondeurs, en causant des explosions inespérées, en remuant les décombres, en détruisant les comportements étanches ${ }^{32}$. Dans le cas présent, notre lecture de la nouvelle «Feliz aniversário » nous a permis d'identifier ces sursauts, ces chocs et ces révélations qui viennent troubler le calme apparent et ce, par le biais de la fête, scène principale de la représentation des liens de famille. Nous avons cherché à démontrer de quelle manière l'espace de la fête pouvait être révélateur tant des relations familiales que sociales, mais s'avérait aussi être un espace privilégié pour comprendre la conception du monde telle que la romancière l'entend, à sa façon, empreinte de mystères, de silences et de regards.

\section{BIBLIOGRAPHIE}

Nadia BATELLA GotLIB, « Un apprentissage des sens » [en ligne], Études Françaises, vol. 25, n 1 (1989), p. 69-80 [disponible le 09/02/2021] <URL : https://www.erudit.org/fr/revues/etudfr/ 1989-v25-n1-etudfr1062/035773ar.pdf>.

Nadia BATELla GotLIB, «Littérature, biographie et photobiographie », Nadia Setti et Maria Graciete Besse (dir.), Clarice Lispector : une pensée en écriture pour notre temps, Paris, L'Harmattan, 2013, p. 31-42.

Jean BAUdRILlaRd et Marc GuILlaume, Figures de l'altérité, Paris, Descartes \& Cie, 1994.

Maria Graciete BESSE, « La créativité nomade », Nadia Setti et Maria Graciete Besse (dir.), Clarice Lispector : une pensée en écriture pour notre temps, Paris, L'Harmattan, 2013, p. 59-78.

Antonio CANDIDO, « No começo era de fato o verbo », Clarice Lispector, A paixão segundo G.H [edição crítica de Benedito Nunes], Brasília, CNPQ, ALCCA, 1988.

Jean CHEVALIER et Alain GHEERBRANT, Dictionnaire des symboles, Paris, Éditions Robert Laffont, Jupiter, 1982.

Roberto CORRÊA DOS SANTOS, Clarice, ela [en ligne], São Paulo, Instituto Moreira Salles, 2012 [disponible le 20/09/2020] <URL : https://claricelispectorims.com.br/wp-content/uploads/ 2016/11/book_34.pdf >.

Natália DE SANTANA GUERELLUS, «La parole est mon domaine sur le monde : circulation et réception de l'œuvre de Clarice Lispector au Portugal » [online], Les Cahiers de Framespa, nº 33 (2020) [disponible le 08/02/21] <URL : http://journals.openedition.org/framespa/7284>. 
Dictionnaire lexicographique du Centre National de Ressources Textuelles et Lexicales (CNRTL)

[disponible le 08/02/2021] <URL :https://www.cnrtl.fr/definition/f\%C3\%AAte>.

Clarice LISPECTOR, A legião estrangeira. Rio de Janeiro, Editora do autor, 1964.

Clarice LISPECTOR, Água viva, Rio de Janeiro, Nova Fronteira, 1980.

Clarice LISPECTOR, A paixão segundo G.H [edição crítica de Benedito Nunes], Brasília, CNPQ, ALCCA, 1988.

Clarice LISPECTOR, Laços de família, Rio de Janeiro, Rocco, 2009.

Clarice LISPECTOR, Liens de famille (traduit par Jacques et Teresa Thiériot), Paris, Édition Des femmes, 1989.

Evando NASCIMENTO, «Les animaux, les choses, la pensée », Nadia Setti et Maria Graciete Besse (dir.) Clarice Lispector : une pensée en écriture pour notre temps, Paris, L'Harmattan, 2013, p. 99-117.

Evando NASCIMENTO, Clarice Lispector: uma literatura pensante, Rio de Janeiro, Civilização Brasileira, 2012.

Benedito NUNES, O drama da linguagem: uma leitura de Clarice Lispector, São Paulo, Editora Ática, 1989

Michel RIAUDEL, «Lire Clarice », Europe, n¹003-1004 (novembre-décembre 2012).

Michel RIAUDEL, « Paradoxes et métamorphoses dans Laços de família de Clarice Lispector » [conférence en ligne], Journée d'études inter-option Agrégation d'Espagnol (mars 2020). [disponible le 08/02/21] <URL : https://www.youtube.com/watch?v=Y1g8IhfOmWc>.

Jean-Claude schмiт, «L'invention de l'anniversaire » [en ligne], Annales. Histoire, Sciences sociales, vol. 62, $\mathrm{n}^{\circ} 4$, (2007) [disponible le 09/02/2021]

<URL : https://www.cairn.info/revue-annales-2007-4-page-793.htm>.

Nadia SETTI et Maria Graciete BESSE (dir.), Clarice Lispector : une pensée en écriture pour notre temps, Paris, L'Harmattan, 2013.

\section{NOTES}

1. Nous traduisons «A ficção de Clarice elaborou um modo de pensar bastante especial ", Evando NASCIMENTO, Clarice Lispector, uma literatura pensante, Rio de Janeiro, Civilização Brasileira, 2012, texte de couverture.

2. Michel RIAUDEL, «Lire Clarice », Europe, n¹003-1004 (novembre-décembre 2012), p. 120.

3. À ce sujet, voir la récente étude de Natália de Santana Guerellus qui analyse la réception et la circulation de l'œuvre de Clarice Lispector à l'échelle internationale: Natália DE SANTANA GUERELLUS, «La parole est mon domaine sur le monde : circulation et réception de l'œuvre de Clarice Lispector au Portugal» [online], Les Cahiers de Framespa, $n^{\circ} 33$ (2020) [disponible le 08/02/21] <URL : http://journals.openedition.org/framespa/7284>

4. « [...] em 1943, a jovem escritora, que surgia a partir do mais completo anonimato, não apenas modificava essencialmente as possibilidades de escrita literária no Brasil, mas obrigava a crítica a rever a sua perspectiva ", Antonio CANDIDO, « No começo era de fato o verbo ", Clarice LISPECTOR, A paixão segundo G.H [edição crítica de Benedito Nunes], Brasília, CNPQ, ALCCA, 1988, p. 19.

5. Naturellement, il existe une multitude d'études scientifiques et théoriques estimables mais qui ne sauraient être citées ici, l'intention de ce travail n'étant pas de dresser la liste exhaustive des travaux portant sur l'œuvre de Clarice Lispector. 
6. Nous reprenons ici les termes employés par Benedito Nunes : « uma temática marcadamente existencial » dans son étude des œuvres de l'auteure. Benedito NUNES, O drama da linguagem: uma leitura de Clarice Lispector, São Paulo, Editora Ática, 1989, p. 100.

7. Pour la présente étude, nous citerons les pages de l'édition brésilienne : Clarice LISPECTOR, Laços de familia, Rio de Janeiro, Rocco, 2009.

8. Nadia BAtella Gotlib, «Un apprentissage des sens » [en ligne], Études Françaises, vol. 25, n 1, 1989, p. 70 (actualisé le 06/04/2006) [disponible le 09/02/2021] <URL : https://www.erudit.org/ fr/revues/etudfr/1989-v25-n1-etudfr1062/035773ar.pdf>

9. «Tensão conflitiva ", " descortínio da existência ", "potência mágica do olhar " sont les concepts développés par Benedito NUNES, op. cit., p. 84-88.

10. «Festa de palavras ». Clarice LISPECTOR, Água viva, Rio de Janeiro, Nova Fronteira, 1980, p. 24.

11. D'après le dictionnaire lexicographique du Centre National de Ressources Textuelle et Lexicales (CNRTL).

12. Maria Graciete BESSE, «La créativité nomade », Nadia Setti et Maria Graciete Besse (dir.), Clarice Lispector : une pensée en écriture pour notre temps, Paris, L'Harmattan, 2013, p. 67.

13. «Chacun à sa façon nous dit que les récits de Clarice Lispector se construisent autour d'une expérience, d'un "événement", qu'ils sont eux-mêmes une expérience, une sorte de rituel invitant à "faire corps", à une sorte de fusion régressive ». Michel RIAUDEL, 2012, op. cit., p. 121.

14. Cette dynamique présente dans les récits de l'auteure est expliquée par Nadia Setti: «Pendant ce temps absolu il y a une vision abrupte du réel, suspension, changement, révolution, mort et résurrection », Nadia SETTI, « Prologue », Nadia Setti et Maria Graciete Besse, op. cit., p. 17. 15. Bien que ces considérations concernent une autre œuvre de la romancière, elles nous semblent pouvoir aussi s'appliquer à la nouvelle «Feliz aniversário » dans la mesure où le narrateur paraît être de connivence avec ses personnages, procédé significatif de l'écriture pensante - ou «pensée-écriture »- de l'auteure.

16. Nadia BATELLA GOTLIB, "Littérature, biographie et photobiographie », Nadia Setti et Maria Graciete Besse, op. cit., p. 31.

17. D'après le dictionnaire des symboles, la symbolique du nom le ramène à la fonction de créateur et présentateur de la chose ou de l'être, étant l'essence et la substance de la manifestation individuelle : «Le nom personnel est bien plus qu'un signe d'identification. Il est une dimension essentielle de l'individu. Le nom étant porteur d'origine, d'appartenance et d'identification à un groupe social tel que celui de la famille par exemple, l'absence de ce dernier engendre la dépersonnalisation et le déracinement de l'individu à quelque milieu qui soit. », Jean CHEVALIER et Alain GHEERBRANT, Dictionnaire des symboles, Paris, Éditions Robert Laffont, Jupiter, 1982, p. 675-676.

18. Jean BAUdRILlaRd et Marc GUILlaume, Figures de l'altérité, Paris, Descartes \& Cie, 1994, p. 23-24.

19. Jean CHEVALIER et Alain GHEERBRANT, op. cit., p. 883.

20. Ibid., p. 803-804.

21. «O descortínio silencioso [...] As coisas exercem uma fascinação contínua sobre os personagens de Clarice Lispector, insinuando-se à experiência interna em momentos de pausa contemplativa, que proporcionam, independentemente do entendimento verbal e discursivo, um saber imediato arraigado à percepção em estado bruto ", Benedito NUNES, op. cit., p. 123.

22. À ce sujet, l'étude de Michel Riaudel précise que le quartier d'Olaria se trouve dans la zone nord de Rio de Janeiro, petite bourgeoisie très modeste, et celui d'Ipanema, dans la zone sud, est le lieu de grande bourgeoisie carioca, du moins à l'époque où Clarice Lispector publie cette nouvelle en 1960. Michel RIAUDEL, «Paradoxes et métamorphoses dans Laços de família de Clarice Lispector » [conférence en ligne], Journée d'études inter-option Agrégation d'Espagnol (mars 2020) [disponible le 08/02/21] <URL : https://www.youtube.com/watch?v=Y1g8IhfOmWc> 
23. Roberto CORRÊA DOS SANTOS, Clarice, ela [en ligne], São Paulo, Instituto Moreira Salles, 2012 [disponible le 20/09/2020] <URL: https://claricelispectorims.com.br/wp-content/uploads/ 2016/11/book_34.pdf >

24. À ce sujet, voir Michel RIAUDEL, op. cit.

25. Benedito NUNES, op. cit., p. 84.

26. À ce sujet, voir l'étude de Evando Nascimento qui propose une réflexion sur la théorie de «l'inquiétante étrangeté » exposée par Freud et qui, appliquée à l'œuvre de Clarice Lispector, conduit à interpréter les relations familiales comme la manifestation du non-familier ou infamilier (Un-heimliche). Evando NAScIMENTO, «Les animaux, les choses, la pensée », Nadia Setti et Maria Graciete Besse, op. cit., p. 103-113.

27. Jean-Claude sснмітт, «L'invention de l'anniversaire» [en ligne], Annales. Histoire, Sciences sociales, vol. 62, $\mathrm{n}^{\circ} 4$, (2007) [disponible le 09/02/2021] <URL: https://www.cairn.info/revueannales-2007-4-page-793.htm>

28. Ce principe est aussi abordé dans le dictionnaire des symboles qui avance que la mort a plusieurs significations : "Libératrice des peines et des soucis, elle n'est pas une fin en soi ; elle ouvre l'accès au règne de l'esprit, à la vie véritable [...] elle est révélation et introduction [...] peut-être la condition d'une vie supérieure à un autre niveau [...]", Jean CHEVALIER et Alain GHEERBRANT, op. cit., p. 650-651.

29. Michel RIAUDEL, 2020, op. cit.

30. « Momento privilegiado sob o aspecto de descortínio da existência [...] esse instante assinala o clímax do desenvolvimento da narrativa ", Benedito NUNES, op. cit., p. 86.

31. Clarice LISPECTOR, "De fundo de gaveta » apud Clarice LISPECTOR, 1988, op. cit., "Dossier da obra », p. 292.

32. Nadia BATELLA GOTLIB, «Littérature, biographie et photobiographie ", Nadia SETTI et Maria Graciete BESSE, op. cit., p. 35.

\section{RÉSUMÉS}

L'intensité de l'écriture de Clarice Lispector réside dans le fait que ses écrits, tous autant qu'ils sont, ne comportent pas d'action à proprement parler mais plutôt des rencontres avec l'autre ou avec soi. Inaugurant ainsi une "littérature pensante » où l'introspection, l'observation, et le langage donnent à son écriture un caractère de confession orale, Clarice Lispector révèle un univers rythmé par un flux de conscience et une quête des sens qui proposent une nouvelle conception du monde, notamment dans la sphère familiale. C'est dans cette optique que peut être lue la nouvelle «Feliz aniversário » dans lequel l'auteure met en scène - non sans ironie - les principaux contours du conflit familial en procédant à une inversion du paradigme de la fête qui devient dès lors un espace empli de significations où les éléments festifs se dissipent pour laisser place à une ambiance oppressante mais révélatrice. Dans le présent article, notre propos sera d'interroger la fonction que tient la fête au centre du récit ainsi que les relations qui y sont dépeintes.

The intensity of Clarice Lispector's writing lies in the fact that her literature do not involve actions, but rather encounters with others or with oneself. Thus, inaugurating a "thinking literature" in which introspection, observation and language give her writing a character of oral 
confessional, Clarice Lispector reveals a universe punctuated by a stream of consciousness and by a quest for the senses which propose a new conception of the world, especially in the family environment. It is in this perspective that the short story "Feliz aniversário" can be read since the author stages - not without irony - the main contours of the family conflicts by proceeding to an inversion of the paradigm of the party. The paradigm of celebration becomes a space filled with meaning in which the festive elements dissipate to give way for an oppressive but revealing atmosphere. In the present article, our purpose will be to question the function of the party at the center of the narrative as well as the relationships that are depicted in "Feliz aniversário".

\section{INDEX}

Mots-clés : Clarice Lispector, Laços de família, Feliz aniversário, fête, observation contemplative, tension conflictuelle

Keywords : Clarice Lispector, Laços de família, Feliz aniversário, celebration, contemplative observation, conflictive tension

\section{AUTEUR}

\section{MIREILLE GARCIA}

Université Rennes 2 